\title{
D-optimal chemical balance weighing designs with autoregressive errors
}

\author{
Krystyna Katulska • Kukasz Smaga
}

Received: 8 July 2011 / Published online: 19 April 2012

(C) The Author(s) 2012. This article is published with open access at Springerlink.com

\begin{abstract}
In this paper, we consider the estimation problem of individual weights of three objects. For the estimation we use the chemical balance weighing design and the criterion of D-optimality. We assume that the error terms $\varepsilon_{i}, i=1,2, \ldots, n$, are a first-order autoregressive process. This assumption implies that the covariance matrix of errors depends on the known parameter $\rho$. We present the chemical balance weighing design matrix $\widetilde{\mathbf{X}}$ and we prove that this design is D-optimal in certain classes of designs for $\rho \in[0,1)$ and it is also D-optimal in the class of designs with the design matrix $\mathbf{X} \in M_{n \times 3}( \pm 1)$ for some $\rho \geq 0$. We prove also the necessary and sufficient conditions under which the design is D-optimal in the class of designs $M_{n \times 3}( \pm 1)$, if $\rho \in[0,1 /(n-2))$. We present also the matrix of the D-optimal factorial design with 3 two-level factors.
\end{abstract}

Keywords Autoregressive process - D-optimal chemical balance weighing design Factorial design $\cdot$ Fischer's inequality $\cdot$ Hadamard's inequality

\section{Introduction}

We make the following assumptions: $n \equiv 0(\bmod 4), \rho \in(-1,1)$ and $M_{n \times m}( \pm 1)$ is the set of all $n \times m$ matrices with each entry $\{-1,1\}$. We wish to estimate the true unknown weights $\theta_{1}, \theta_{2}, \theta_{3}$ of 3 objects employing $n$ measuring operations using a chemical balance. Denote the observations in these $n$ operations by $y_{1}, y_{2}, \ldots, y_{n}$,

\footnotetext{
K. Katulska · Ł. Smaga $(\varangle)$

Faculty of Mathematics and Computer Science, Adam Mickiewicz University, ul. Umultowska 87, 61-614 Poznań, Poland

e-mail: 1s@amu.edu.pl

K. Katulska

e-mail: krakat@amu.edu.pl
} 
respectively. Let observations follow the linear model

$$
\mathbf{Y}=\mathbf{X} \boldsymbol{\theta}+\boldsymbol{\varepsilon}
$$

where $\mathbf{Y}=\left[y_{1}, y_{2}, \ldots, y_{n}\right]^{\prime}$ is an $n \times 1$ column vector of observations, $\boldsymbol{\theta}=\left[\theta_{1}, \theta_{2}, \theta_{3}\right]^{\prime}$ is the vector of true unknown parameters, the matrix

$$
\mathbf{X}=\left[\begin{array}{ccc}
x_{11} & x_{12} & x_{13} \\
x_{21} & x_{22} & x_{23} \\
\vdots & \vdots & \vdots \\
x_{n 1} & x_{n 2} & x_{n 3}
\end{array}\right]
$$

is called the design matrix and $x_{i j}=-1$ if the $j$ th object is placed on the left pan or $x_{i j}=1$ if the $j$ th object is placed on the right pan during the $i$ th weighing operation, the vector $\varepsilon=\left[\varepsilon_{1}, \varepsilon_{2}, \ldots, \varepsilon_{n}\right]^{\prime}$ is the so-called vector of error components. We assume that the sequence $\left(\varepsilon_{i}\right)$ is a first-order autoregressive process. Therefore $E(\boldsymbol{\varepsilon})=[0,0, \ldots, 0]^{\prime}$ is an $n \times 1$ of zeros and $\operatorname{Var}(\boldsymbol{\varepsilon})=\frac{1}{1-\rho^{2}} \mathbf{S}$, where

$$
\mathbf{S}=\left[\begin{array}{cccccc}
1 & \rho & \rho^{2} & \cdots & \rho^{n-2} & \rho^{n-1} \\
\rho & 1 & \rho & \cdots & \rho^{n-3} & \rho^{n-2} \\
\rho^{2} & \rho & 1 & \cdots & \rho^{n-4} & \rho^{n-3} \\
\vdots & \vdots & \vdots & \ddots & \vdots & \vdots \\
\rho^{n-2} & \rho^{n-3} & \rho^{n-4} & \cdots & 1 & \rho \\
\rho^{n-1} & \rho^{n-2} & \rho^{n-3} & \cdots & \rho & 1
\end{array}\right]
$$

and $-1<\rho<1$. If the design matrix $\mathbf{X}$ is full column $\operatorname{rank}$, i.e. $\operatorname{rank}(\mathbf{X})=3$, then the generalized least-squares estimator of vector $\boldsymbol{\theta}$ is given by $\hat{\boldsymbol{\theta}}=\left(\mathbf{X}^{\prime} \mathbf{S}^{-1} \mathbf{X}\right)^{-1} \mathbf{X}^{\prime} \mathbf{S}^{-1} \mathbf{Y}$ and $\operatorname{Var}(\hat{\boldsymbol{\theta}})=\frac{1}{1-\rho^{2}}\left(\mathbf{X}^{\prime} \mathbf{S}^{-1} \mathbf{X}\right)^{-1}$.

Definition 1 The design with the design matrix $\mathbf{X}_{D}$ is D-optimal in the class of designs with the design matrix in the set $\mathcal{C} \subseteq M_{n \times 3}( \pm 1)$ if

$$
\operatorname{det}\left(\mathbf{X}_{D}^{\prime} \mathbf{S}^{-1} \mathbf{X}_{D}\right)=\max \left\{\operatorname{det}\left(\mathbf{X}^{\prime} \mathbf{S}^{-1} \mathbf{X}\right): \mathbf{X} \in \mathcal{C}\right\}
$$

The matrix $\frac{1}{1-\rho^{2}} \mathbf{A}$ is the inverse matrix of $\mathbf{S}$, where

$$
\mathbf{A}=\left[\begin{array}{cccccc}
1 & -\rho & 0 & \cdots & 0 & 0 \\
-\rho & 1+\rho^{2} & -\rho & \cdots & 0 & 0 \\
0 & -\rho & 1+\rho^{2} & \cdots & 0 & 0 \\
\vdots & \vdots & \vdots & \ddots & \vdots & \vdots \\
0 & 0 & 0 & \cdots & 1+\rho^{2} & -\rho \\
0 & 0 & 0 & \cdots & -\rho & 1
\end{array}\right]
$$

The matrix $\mathbf{A}$ is positive definite for $\rho \in(-1,1)$. 
Lemma 1 Let $\mathbf{A}$ be given by (2). The design $\mathbf{X}_{D}$ is D-optimal in the class of designs with design matrices in $\mathcal{C} \subseteq M_{n \times 3}( \pm 1)$ if

$$
\operatorname{det}\left(\mathbf{X}_{D}^{\prime} \mathbf{A} \mathbf{X}_{D}\right)=\max \left\{\operatorname{det}\left(\mathbf{X}^{\prime} \mathbf{A} \mathbf{X}\right): \mathbf{X} \in \mathcal{C}\right\}
$$

The case $\rho=0$ is well known in the literature. The D-optimality problem is considered for example in Hadamard (1893), Cohn (1967, 1989), Galil and Kiefer (1980), Cheng (1980) or Jacroux et al. (1983). For $\rho \neq 0$, the problem of the D-optimal design was considered in Li and Yang (2005), Yeh and Lo Huang (2005) and Katulska and Smaga (2010). In these papers, the D-optimality problem was solved in some subclasses of $M_{n \times 3}( \pm 1)$.

We show in Sect. 2 that the design with the design matrix

$$
\widetilde{\mathbf{X}}^{\prime}=\left\{\begin{array}{c}
{\left[\begin{array}{c}
<n>^{+} \\
<n / 2>^{+}<n / 2>^{-} \\
<n / 4>^{+}<n / 2>^{+}<n / 4>^{-}
\end{array}\right] \quad \text { if } \quad \frac{n}{4}=2 k-1} \\
{\left[\begin{array}{c}
<n>^{+} \\
<n / 2>^{+}<n / 2>^{-} \\
<n / 4>^{+}<n / 2>^{-}<n / 4>^{+}
\end{array}\right] \quad \text { if } \quad \frac{n}{4}=2 k}
\end{array}\right.
$$

where

$$
\begin{aligned}
& <t>^{+}=\left[(-1)^{2},(-1)^{3},(-1)^{4}, \ldots,(-1)^{t+1}\right], \\
& <t>^{-}=\left[(-1)^{1},(-1)^{2},(-1)^{3}, \ldots,(-1)^{t}\right]
\end{aligned}
$$

and $k=1,2, \ldots$, is D-optimal in certain classes of designs for $\rho \in[0,1)$ and this design is D-optimal in the class of designs with design matrices $\mathbf{X} \in M_{n \times 3}( \pm 1)$ for $\rho \in[0,1 /(n-2)]$. We present also theorems giving necessary and sufficient conditions under which the design for three objects is D-optimal. At the end of Sect. 2, we compare our results with results in Yeh and Lo Huang (2005). In Sect. 3, we present the matrix of the D-optimal factorial design with 3 two-level factors, which is constructed by the matrix of D-optimal weighing design for three objects. In the appendix, we give some lemmas and theorems, which contain properties used in proofs of main results in this paper, and present the proofs of theorems from Sect. 2 and 3.

\section{D-optimal weighing design when $\rho \in[0,1)$}

We must introduce some notation, wchich we use in this paper. We define for $\mathbf{x}=$ $\left[x_{1}, x_{2}, \ldots, x_{n}\right]^{\prime} \in M_{n \times 1}( \pm 1)$ the following numbers

$$
\begin{aligned}
& \operatorname{cons}(\mathbf{x})=\#\left\{i: x_{i}=x_{i+1}, 1 \leq i \leq n-1\right\} \\
& \operatorname{fcons}(\mathbf{x})=\min \left\{i: x_{i}=x_{i+1}, 1 \leq i \leq n-1\right\}, \\
& \operatorname{scons}(\mathbf{x})=\min \left\{i: i>\operatorname{fcons}(\mathbf{x}), x_{i}=x_{i+1}, 1 \leq i \leq n-1\right\} .
\end{aligned}
$$


For example, if $\mathbf{x}=[1,-1,1,1,-1,1,-1,-1,1,-1,1,-1]^{\prime}$, then cons $(\mathbf{x})=$ 2, fcons $(\mathbf{x})=3, \operatorname{scons}(\mathbf{x})=7$. For the matrix $\widetilde{\mathbf{X}}$ given by (3) from Lemmas 4 and 5 , we have

$$
\begin{aligned}
\operatorname{det}\left(\widetilde{\mathbf{X}}^{\prime} \mathbf{A} \widetilde{\mathbf{X}}\right) & =\operatorname{det}\left[\begin{array}{ccc}
\Delta & 0 & -2 \rho(1+\rho) \\
0 & \Delta-4 \rho & 0 \\
-2 \rho(1+\rho) & 0 & \Delta-8 \rho
\end{array}\right] \\
& =\Delta(\Delta-4 \rho)(\Delta-8 \rho)-4 \rho^{2}(1+\rho)^{2}(\Delta-4 \rho),
\end{aligned}
$$

where $\Delta=\widetilde{\mathbf{x}}^{\prime} \mathbf{A} \widetilde{\mathbf{x}}=(n-2)(1+\rho)^{2}+2(1+\rho)$ and $\widetilde{\mathbf{x}}$ is the first column of the matrix $\widetilde{\mathbf{X}}$. In the following theorem, we prove that the design $\widetilde{\mathbf{X}}$ given by (3) is D-optimal in some large subclass of $M_{n \times 3}( \pm 1)$ for all $\rho \in[0,1)$.

Theorem 1 If $n \equiv 0(\bmod 4)$ and $\rho \in[0,1)$, then the design with the design matrix $\widetilde{\mathbf{X}}$ given by (3) is D-optimal in the class of designs with design matrices $\mathbf{X}=[\mathbf{x}, \mathbf{y}, \mathbf{z}] \in$ $C_{1} \cup C_{2} \cup C_{3}$, $\operatorname{rank}(\mathbf{X})=3$, where

$$
\begin{aligned}
& C_{1}=\left\{[\boldsymbol{\alpha}, \boldsymbol{\beta}, \boldsymbol{\gamma}] \in M_{n \times 3}( \pm 1): \operatorname{cons}(\boldsymbol{\alpha})=1, \operatorname{cons}(\boldsymbol{\beta})=1, \operatorname{cons}(\boldsymbol{\gamma})=1\right\}, \\
& C_{2}=\left\{[\boldsymbol{\alpha}, \boldsymbol{\beta}, \boldsymbol{\gamma}] \in M_{n \times 3}( \pm 1): \operatorname{cons}(\boldsymbol{\alpha}) \geq 1, \operatorname{cons}(\boldsymbol{\beta}) \geq 1, \operatorname{cons}(\boldsymbol{\gamma}) \geq 2\right\}, \\
& C_{3}=\left\{[\boldsymbol{\alpha}, \boldsymbol{\beta}, \boldsymbol{\gamma}] \in M_{n \times 3}( \pm 1): \operatorname{cons}(\boldsymbol{\alpha})=0, \operatorname{cons}(\boldsymbol{\beta}) \geq 1, \operatorname{cons}(\boldsymbol{\gamma}) \geq 2\right\}
\end{aligned}
$$

The proof of Theorem 1 is given in the "Appendix".

In the following theorem, we consider the whole class of designs $M_{n \times 3}( \pm 1)$ and we show that the design $\widetilde{\mathbf{X}}$ of the form (3) is still D-optimal in this class if $\rho \in$ $[0,1 /(n-2)]$.

Theorem 2 If $n \equiv 0(\bmod 4)$ and $\rho \in[0,1 /(n-2)]$, then the design with the design matrix $\widetilde{\mathbf{X}}$ given by (3) is D-optimal in the class of designs with design matrices $\mathbf{X}=[\mathbf{x}, \mathbf{y}, \mathbf{z}] \in M_{n \times 3}( \pm 1)$ and $\operatorname{rank}(\mathbf{X})=3$.

The proof of Theorem 2 is given in the "Appendix".

We now formulate the necessary and sufficient conditions under which the design is D-optimal in $M_{n \times 3}( \pm 1)$.

Theorem 3 Let $n \equiv 0(\bmod 4)$ and $\rho \in[0,1 /(n-2))$. If $\mathbf{X}_{*}=\left[\mathbf{x}_{*}, \mathbf{y}_{*}, \mathbf{z}_{*}\right] \in$ $M_{n \times 3}( \pm 1)$ and $\operatorname{rank}\left(\mathbf{X}_{*}\right)=3$, then the design $\mathbf{X}_{*}$ is D-optimal in the class of designs $\mathbf{X} \in M_{n \times 3}( \pm 1), \operatorname{rank}(\mathbf{X})=3$ if and only if

$$
\mathbf{X}_{*}^{\prime} \mathbf{A X}_{*}=\left[\begin{array}{ccc}
\Delta & 0 & \pm 2 \rho(1+\rho) \\
0 & \Delta-4 \rho & 0 \\
\pm 2 \rho(1+\rho) & 0 & \Delta-8 \rho
\end{array}\right]
$$

exact to permuting columns of the matrix $\mathbf{X}_{*}$.

The proof of Theorem 3 is given in the "Appendix". 
Theorem 4 Let $n \equiv 0(\bmod 4), \rho \in(0,1 /(n-2))$ and $\mathbf{X}_{*}=\left[\mathbf{x}_{*}, \mathbf{y}_{*}, \mathbf{z}_{*}\right] \in$ $M_{n \times 3}( \pm 1), \operatorname{rank}\left(\mathbf{X}_{*}\right)=3$. The design $\mathbf{X}_{*}$ is D-optimal in the class of designs $\mathbf{X} \in M_{n \times 3}( \pm 1), \operatorname{rank}(\mathbf{X})=3$ if and only if

$$
\mathbf{X}_{*} \in\left\{[\boldsymbol{\alpha}, \boldsymbol{\beta}, \boldsymbol{\gamma}] \in M_{n \times 3}( \pm 1): \operatorname{cons}(\boldsymbol{\alpha})=0, \operatorname{cons}(\boldsymbol{\beta})=1, \operatorname{cons}(\boldsymbol{\gamma})=2\right\}
$$

and fcons $\left(\mathbf{y}_{*}\right)=\frac{n}{2}$, fcons $\left(\mathbf{z}_{*}\right)=\frac{n}{4}, \operatorname{scons}\left(\mathbf{z}_{*}\right)=\frac{3 n}{4}$ exact to permuting columns of the matrix $\mathbf{X}_{*}$.

The proof of Theorem 4 is given in the "Appendix".

Yeh and Lo Huang (2005) considered the exact D-optimal designs with 2 two-level factors and $n$ autocorrelated observations. They proved that the design with the design matrix

$$
\hat{\mathbf{X}}^{\prime}=\left[\begin{array}{c}
\mathbf{1}^{\prime} \\
<n>^{+} \\
<n / 2>^{+}<n / 2>^{-}
\end{array}\right]
$$

where $\mathbf{1}=[1,1, \ldots, 1] \in M_{n \times 1}( \pm 1)$, is D-optimal in considered class of designs for $\rho \in(0,1)$. But as it is described in Banerjee (1975), the design from Yeh and Lo Huang (2005) can be used as the design for finding the weights of three objects. It is easy to calculate that

$$
\forall_{\rho \in(0,1)} \operatorname{det}\left(\widetilde{\mathbf{X}}^{\prime} \mathbf{A} \tilde{\mathbf{X}}\right)>\operatorname{det}\left(\hat{\mathbf{X}}^{\prime} \mathbf{A} \hat{\mathbf{X}}\right)
$$

so the design $\hat{\mathbf{X}}$ given by (5) is D-worse than the design $\widetilde{\mathbf{X}}$ given by (3). Therefore, the D-optimal design from the subclass of designs with the first column of all ones is not D-optimal in the set $M_{n \times 3}( \pm 1)$.

\section{D-optimal $2^{3}$ factorial design}

In this section, we consider designs of 3 factorial main effects with a first-order autoregressive errors. We describe the model similarly to Cheng (1980). Suppose we have 3 factors each with two levels 0 and 1 . For any observation $y_{j_{1} j_{2} j_{3}}$ on the $j_{i}$ th level of factor $i, i=1,2,3, j_{i}=0$ or $1, E\left(y_{j_{1} j_{2} j_{3}}\right)$ can be written as

$$
E\left(y_{j_{1} j_{2} j_{3}}\right)=\phi^{0}+\sum_{i=1}^{3} x_{i} \phi_{i}^{1}
$$

where $\phi^{0}$ (grand mean) and $\phi_{i}^{1}, i=1,2,3$ (main effects) are unknown parameters and $x_{i}=1(-1)$ if $j_{i}=1(0)$. The sequence $\epsilon_{i}$ of error terms is a first-order autoregressive process. In this setting, the design matrix $\mathbf{X} \in M_{n \times 4}( \pm 1)$ has the first column of all ones. Then D-optimality criterion is defined similarly to Definition 1 . In this model, we proved the following theorem. 
Theorem 5 Let $n \equiv 0(\bmod 4)$ and $\rho \in(0,1 /(n-2))$. If $\mathbf{X}^{*}$ is the matrix of the D-optimal weighing design for three objects, then the design with the matrix $\left[\mathbf{1}, \mathbf{X}^{*}\right]$ is D-optimal factorial design with 3 two-level factors with a first-order autoregressive errors.

The proof of Theorem 5 is very long and similar to the proofs of Theorems 1, 2 and 3 , so we present only the sketch of it in the "Appendix".

Acknowledgments Authors are thankful to the referees for their constructive suggestions which improved the presentation of this article.

Open Access This article is distributed under the terms of the Creative Commons Attribution License which permits any use, distribution, and reproduction in any medium, provided the original author(s) and the source are credited.

\section{Appendix}

First, we give several well known results.

Lemma 2 Let $\mathbf{X}$ be an $n \times m$ matrix and $\mathbf{B}$ be an $n \times n$ positive definite matrix. Then matrix $\mathbf{X}^{\prime} \mathbf{B X}$ is positive semi-definite and $\operatorname{rank}\left(\mathbf{X}^{\prime} \mathbf{B X}\right)=\operatorname{rank}(\mathbf{X})$. Moreover, $\mathbf{X}^{\prime} \mathbf{B X}$ is positive definite if and only if a matrix $\mathbf{X}$ is of full column rank.

Theorem 6 (Hadamard's inequality) Assume that $\mathbf{P}=\left[p_{i j}\right]$ is an $n \times n$ positive semi-definite. Then

$$
\operatorname{det}(\mathbf{P}) \leq \prod_{i=1}^{n} p_{i i}
$$

Futhermore, when $\mathbf{P}$ is positive definite, then the equality holds if and only if $\mathbf{P}$ is diagonal.

Theorem 7 (Fischer's inequality) If a positive definite matrix

$$
\mathbf{P}=\left[\begin{array}{cc}
\mathbf{B} & \mathbf{C} \\
\mathbf{C}^{\prime} & \mathbf{D}
\end{array}\right]
$$

is partitioned so that $\mathbf{B}$ and $\mathbf{D}$ are square and nonempty, then $\operatorname{det}(\mathbf{P}) \leq \operatorname{det}(\mathbf{B}) \operatorname{det}(\mathbf{D})$ and the equality holds if and only if $\mathbf{C}=\mathbf{0}$.

Now, we present some simple lemmas.

Lemma 3 Let $\mathbf{X} \in M_{n \times 3}( \pm 1)$. Then the determinant of the matrix $\mathbf{X}^{\prime} \mathbf{A X}$, where $\mathbf{A}$ is given by (2), does not change if we interchange two columns of the matrix $\mathbf{X}$ or we multiply any column of this matrix by -1 .

Proof This Lemma follows from properties of determinants. 
Lemma 4 If $\rho \neq 0, \Delta=\widetilde{\mathbf{x}}^{\prime} \mathbf{A} \widetilde{\mathbf{x}}=(n-2)(1+\rho)^{2}+2(1+\rho)$, where $\widetilde{\mathbf{x}}$ is the first column of the matrix $\widetilde{\mathbf{X}}$ given by (3), $n \equiv 0(\bmod 4), \mathbf{x} \in M_{n \times 1}( \pm 1)$ and $\xi=0,1,2, \ldots, n-1$, then $\operatorname{cons}(\mathbf{x})=\xi$ if and only if $\mathbf{x}^{\prime} \mathbf{A} \mathbf{x}=\Delta-4 \xi \rho$.

Proof Let $\mathbf{x}=\left[x_{1}, x_{2}, \ldots, x_{n}\right]^{\prime}$. The lemma follows from the equality (see the proof of Lemma 5 in Yeh and Lo Huang 2005)

$$
\mathbf{x}^{\prime} \mathbf{A} \mathbf{x}=(n-2)\left(1+\rho^{2}\right)+2-2 \rho\left(x_{1} x_{2}+x_{2} x_{3}+\cdots+x_{n-1} x_{n}\right) .
$$

Lemma 5 Let $\mathbf{x}, \mathbf{y} \in M_{n \times 1}( \pm 1)$ and $n \equiv 0(\bmod 4)$.

(a) If $\operatorname{cons}(\mathbf{x})=\operatorname{cons}(\mathbf{y})=1$, fcons $(\mathbf{x})=a>b=$ fcons $(\mathbf{y})$, then

$$
\mathbf{x}^{\prime} \mathbf{A y}=\left\{\begin{array}{ll}
(n-2 a+2 b-2)(1+\rho)^{2}+2(1+\rho) & \text { if } x_{1}=y_{1} \\
-\left((n-2 a+2 b-2)(1+\rho)^{2}+2(1+\rho)\right) & \text { if } x_{1} \neq y_{1}
\end{array} .\right.
$$

(b) If $\operatorname{cons}(\mathbf{x})=0, \operatorname{cons}(\mathbf{y})=2, a=\mathrm{fcons}(\mathbf{y}), b=\operatorname{scons}(\mathbf{y})$, then

$$
\mathbf{x}^{\prime} \mathbf{A y}=\left\{\begin{array}{ll}
(n+2 a-2 b-2)(1+\rho)^{2}+2(1+\rho) & \text { if } x_{1}=y_{1} \\
-\left((n+2 a-2 b-2)(1+\rho)^{2}+2(1+\rho)\right) & \text { if } x_{1} \neq y_{1}
\end{array} .\right.
$$

(c) If $\operatorname{cons}(\mathbf{x})=0, \operatorname{cons}(\mathbf{y})=1, a=\mathrm{fcons}(\mathbf{y})$, then

$$
\mathbf{x}^{\prime} \mathbf{A y}=\left\{\begin{array}{ll}
(2 a-n)(1+\rho)^{2} & \text { if } x_{1}=y_{1} \\
-(2 a-n)(1+\rho)^{2} & \text { if } x_{1} \neq y_{1}
\end{array} .\right.
$$

(d) If $\operatorname{cons}(\mathbf{x})=1$, fcons $(\mathbf{x})=\frac{n}{2}, \operatorname{cons}(\mathbf{y})=2, b=\operatorname{fcons}(\mathbf{y})<\frac{n}{2}, c=\operatorname{scons}(\mathbf{y})>$ $\frac{n}{2}$, then

$$
\mathbf{x}^{\prime} \mathbf{A y}=\left\{\begin{array}{ll}
2(b+c-n)(1+\rho)^{2} & \text { if } x_{1}=y_{1} \\
-2(b+c-n)(1+\rho)^{2} & \text { if } x_{1} \neq y_{1}
\end{array} .\right.
$$

Proof For example, we prove $(c)$, when $x_{1}=y_{1}=1$. If $a$ is odd, then $y_{a}=1$, else $y_{a}=-1$. In both these situations, we have

$$
\begin{aligned}
\mathbf{y}^{\prime} \mathbf{A} \mathbf{x} & =\mathbf{y}^{\prime}\left[1+\rho,-(1+\rho)^{2},(1+\rho)^{2}, \ldots,-(1+\rho)^{2},(1+\rho)^{2},-(1+\rho)\right]^{\prime} \\
& =(2 a-n)(1+\rho)^{2} .
\end{aligned}
$$

Lemma 6 Assume that $\rho \in(0,1)$ and $a=2 k, k \in Z$. Then $(a(1+\rho)+2)^{2} \geq 4 \rho^{2}$. 
Proof It is easy to see that

$$
\begin{aligned}
(2 k(1+\rho)+2)^{2}-4 \rho^{2} & =(2 k(1+\rho)+2-2 \rho)(2 k(1+\rho)+2+2 \rho) \\
& =4(k+k \rho+1-\rho)(k+k \rho+1+\rho) \\
& =4(k+1)(1+\rho)(k+1+\rho(k-1)) \geq 0,
\end{aligned}
$$

because

$$
\begin{aligned}
& \text { if } k \geq 0 \text {, then }(k+1)(1+\rho)>0 \text { and } k+1+\rho(k-1)>0, \\
& \text { if } k<0 \text {, then }(k+1)(1+\rho) \leq 0 \text { and } k+1+\rho(k-1) \leq 0 .
\end{aligned}
$$

Lemma 7 Let $\rho \in(0,1)$. The function $g: R \rightarrow R$, $g(t)=(t(1+\rho)+2)^{2}$ is increasing for $t \geq \frac{-2}{1+\rho}$ and decreasing for $t \leq \frac{-2}{1+\rho}$.

Proof This is clear because the function $g$ is the quadratic function with zero in $t_{0}=\frac{-2}{1+\rho}$ for fixed $\rho \in(0,1)$.

Now, we present proofs of the theorems.

Proof of Theorem 1 If $\rho=0$, then the matrix $\mathbf{S}$ given by (1) is equal to the identity matrix and from Hadamard (1893) we have $\operatorname{det}\left(\widetilde{\mathbf{X}}^{\prime} \mathbf{S}^{-1} \widetilde{\mathbf{X}}\right)=\operatorname{det}\left(\widetilde{\mathbf{X}} \widetilde{\mathbf{X}}^{\prime}\right) \geq \operatorname{det}\left(\mathbf{X}^{\prime} \mathbf{X}\right)=$ $\operatorname{det}\left(\mathbf{X}^{\prime} \mathbf{S}^{-1} \mathbf{X}\right)$, because $\widetilde{\mathbf{X}}^{\prime} \widetilde{\mathbf{X}}=n \mathbf{I}$, where $\mathbf{I}$ is the identity matrix of size 3 . Therefore, we assume that $\rho \in(0,1)$. From Lemma 3 , we suppose that the first row of the matrix $\mathbf{X}$ is $[1,1,1]$. The matrix $\mathbf{X}^{\prime} \mathbf{A} \mathbf{X}$ is positive definite from Lemma 2, because the matrix $\mathbf{A}$ given by (2) is positive definite and $\operatorname{rank}(\mathbf{X})=3$.

We must consider three cases. We show in Case $i, i=1,2,3$, that

$$
\operatorname{det}\left(\widetilde{\mathbf{X}}^{\prime} \mathbf{A} \widetilde{\mathbf{X}}\right) \geq \operatorname{det}\left(\mathbf{X}^{\prime} \mathbf{A X}\right)
$$

for all $\mathbf{X} \in C_{i}$, and by Lemma 1 , we conclude that the matrix $\widetilde{\mathbf{X}}$ given by (3) is the matrix of the D-optimal design in the considered class of the designs.

Case $1 \mathbf{X}=[\mathbf{x}, \mathbf{y}, \mathbf{z}] \in C_{1}$

From Lemma 3, we can assume that $c=\operatorname{fcons}(\mathbf{z})<b=\mathrm{fcons}(\mathbf{y})<a=\mathrm{fcons}(\mathbf{x})$. By definition of fcons $(\mathbf{x})$, we obtain $c \geq 1$ and $a \leq n-1$. If $n=4$, then there is only one design with the design matrix such that $c=1, b=2, a=3$. So $\operatorname{det}\left(\mathbf{X}^{\prime} \mathbf{A X}\right)=$ $-32 \rho^{3}+32, \operatorname{det}\left(\widetilde{\mathbf{X}}^{\prime} \mathbf{A} \widetilde{\mathbf{X}}\right)=32 \rho^{3}+64 \rho^{2}+96 \rho+64$. Hence $\operatorname{det}\left(\widetilde{\mathbf{X}}^{\prime} \mathbf{A} \widetilde{\mathbf{X}}\right)-\operatorname{det}\left(\mathbf{X}^{\prime} \mathbf{A X}\right)=$ $32\left(2 \rho^{3}+2 \rho^{2}+3 \rho+1\right)>0$. If $n \geq 8$, then from Lemmas 4 and 5(a), it follows that

$$
\operatorname{det}\left(\mathbf{X}^{\prime} \mathbf{A X}\right)=\operatorname{det}\left[\begin{array}{ccc}
\Delta-4 \rho & \Gamma_{1} & \Gamma_{2} \\
\Gamma_{1} & \Delta-4 \rho & \Gamma_{3} \\
\Gamma_{2} & \Gamma_{3} & \Delta-4 \rho
\end{array}\right],
$$

where

$$
\begin{aligned}
& \Gamma_{1}=(n-2 a+2 b-2)(1+\rho)^{2}+2(1+\rho), \\
& \Gamma_{2}=(n-2 a+2 c-2)(1+\rho)^{2}+2(1+\rho),
\end{aligned}
$$




$$
\Gamma_{3}=(n-2 b+2 c-2)(1+\rho)^{2}+2(1+\rho) .
$$

There are many posible design matrices $\mathbf{X}$ which depend on the numbers $a, b$ and $c$. Unfortunately, we do not know one proof of the inequality (6) for all of them. Therefore, we must divide the proof into subcases.

Subcase $1.1 a \leq \frac{n}{2}$ or $\left(a>\frac{n}{2}\right.$ and $\left.b \geq a-\frac{n}{2}+2\right)$. From Fischer's inequality, we have

$$
\operatorname{det}\left(\mathbf{X}^{\prime} \mathbf{A X}\right) \leq(\Delta-4 \rho)\left[(\Delta-4 \rho)^{2}-\Gamma_{1}^{2}\right]
$$

Since $n-2 a+2 b-2 \geq 2$ and $\frac{-2}{1+\rho} \in(-2,-1)$, we conclude from Lemma 7 that the inequality $\Gamma_{1}^{2} \geq\left[2(1+\rho)^{2}+2(1+\rho)\right]^{2}$ holds. Therefore by inequality (8), we obtain

$$
\operatorname{det}\left(\mathbf{X}^{\prime} \mathbf{A X}\right) \leq(\Delta-4 \rho)\left[(\Delta-4 \rho)^{2}-\left[2(1+\rho)^{2}+2(1+\rho)\right]^{2}\right]
$$

Hence

$$
\begin{aligned}
\operatorname{det}\left(\widetilde{\mathbf{X}}^{\prime} \mathbf{A} \widetilde{\mathbf{X}}\right)-\operatorname{det}\left(\mathbf{X}^{\prime} \mathbf{A X}\right) \geq & \operatorname{det}\left(\widetilde{\mathbf{X}}^{\prime} \mathbf{A} \widetilde{\mathbf{X}}\right)-(\Delta-4 \rho)\left[(\Delta-4 \rho)^{2}\right. \\
& \left.-\left[2(1+\rho)^{2}+2(1+\rho)\right]^{2}\right] \\
= & (\Delta-4 \rho)\left[16 \rho^{3}+32 \rho^{2}+48 \rho+16\right]>0 .
\end{aligned}
$$

Subcase $1.2 a>\frac{n}{2}$ and $b \leq a-\frac{n}{2}$. Therefore, $b \leq \frac{n}{2}-1$ and $n-2 b+2 c-2 \geq 2 c \geq 2$ because $c \geq 1$. From Fischer's inequality and Lemma 7, it follows that

$$
\begin{aligned}
\operatorname{det}\left(\mathbf{X}^{\prime} \mathbf{A X}\right) & \leq(\Delta-4 \rho)\left[(\Delta-4 \rho)^{2}-\Gamma_{3}^{2}\right] \\
& \leq(\Delta-4 \rho)\left[(\Delta-4 \rho)^{2}-\left[2(1+\rho)^{2}+2(1+\rho)\right]^{2}\right]
\end{aligned}
$$

Thus, applying similar arguments to those in the proof of Subcase 1.1, we obtain (6).

Subcase $1.3 a>\frac{n}{2}, b=a-\frac{n}{2}+1$ and $c \neq b-\frac{n}{2}+1$. Thus $b \leq \frac{n}{2}$ and $\left(c \leq b-\frac{n}{2}\right.$ or $\left.c \geq b-\frac{n}{2}+2\right)$. But $c \leq b-\frac{n}{2} \leq 0$ and therefore we have only the case $c \geq b-\frac{n}{2}+2$. Thus $n-2 b+2 c-2 \geq 2$ and the rest of the proof runs as in Subcase 1.2.

Subcase $1.4 a>\frac{n}{2}, b=a-\frac{n}{2}+1$ and $c=b-\frac{n}{2}+1$. In this subcase $c=a-n+2 \leq 1$ but $c \geq 1$ so $c=1$. Therefore $b=\frac{n}{2}, a=n-1$ and by (7), we have $\Gamma_{1}=\Gamma_{3}=$ 


$$
\begin{aligned}
2(1+\rho), \Gamma_{2}=(2-n)(1+\rho)^{2} & +2(1+\rho) \text { and } \\
\operatorname{det}\left(\widetilde{\mathbf{X}}^{\prime} \mathbf{A} \widetilde{\mathbf{X}}\right)-\operatorname{det}\left(\mathbf{X}^{\prime} \mathbf{A X}\right)= & \operatorname{det}\left(\widetilde{\mathbf{X}}^{\prime} \mathbf{A} \widetilde{\mathbf{X}}\right)-(\Delta-4 \rho)^{3} \\
& -8(1+\rho)^{3}((2-n)(1+\rho)+2) \\
& +(\Delta-4 \rho)(1+\rho)^{2}((2-n)(1+\rho)+2)^{2} \\
& +8(\Delta-4 \rho)(1+\rho)^{2} \\
= & (\Delta-4 \rho)\left[-16 \rho^{2}-4 \rho^{2}(1+\rho)^{2}\right. \\
& +(1+\rho)^{2}((2-n)(1+\rho)+2)^{2} \\
& \left.+8(1+\rho)^{2}\right]-8(1+\rho)^{3}((2-n)(1+\rho)+2) .
\end{aligned}
$$

From Lemma 7, we obtain $((2-n)(1+\rho)+2)^{2} \geq(-4(1+\rho)+2)^{2}$. Hence

$$
\begin{aligned}
\operatorname{det}\left(\tilde{\mathbf{X}}^{\prime} \mathbf{A} \widetilde{\mathbf{X}}\right)-\operatorname{det}\left(\mathbf{X}^{\prime} \mathbf{A X}\right) \geq & (\Delta-4 \rho)\left[-16 \rho^{2}-4 \rho^{2}(1+\rho)^{2}\right. \\
& +(1+\rho)^{2}(-4(1+\rho)+2)^{2} \\
& \left.+8(1+\rho)^{2}\right]-8(1+\rho)^{3}((2-n)(1+\rho)+2) \\
= & (\Delta-4 \rho)\left[4\left(3 \rho^{4}+10 \rho^{3}+8 \rho^{2}+6 \rho+1\right)\right. \\
& \left.+8(1+\rho)^{2}\right]-8(1+\rho)^{3}((2-n)(1+\rho)+2)>0,
\end{aligned}
$$

because $(2-n)(1+\rho)+2<0$.

Case $2 \mathbf{X}=[\mathbf{x}, \mathbf{y}, \mathbf{z}] \in C_{2}$

In this case we use Hadamard's inequality. From this inequality, we obtain

$$
\operatorname{det}\left(\mathbf{X}^{\prime} \mathbf{A X}\right)=\operatorname{det}\left([\mathbf{x}, \mathbf{y}, \mathbf{z}]^{\prime} \mathbf{A}[\mathbf{x}, \mathbf{y}, \mathbf{z}]\right) \leq\left(\mathbf{x}^{\prime} \mathbf{A} \mathbf{x}\right)\left(\mathbf{y}^{\prime} \mathbf{A y}\right)\left(\mathbf{z}^{\prime} \mathbf{A z}\right)
$$

The inequalities $\mathbf{x}^{\prime} \mathbf{A x} \leq \Delta-4 \rho, \mathbf{y}^{\prime} \mathbf{A y} \leq \Delta-4 \rho$ and $\mathbf{z}^{\prime} \mathbf{A} \mathbf{z} \leq \Delta-8 \rho$ hold by Lemma 4. Hence

$$
\begin{aligned}
\operatorname{det}\left(\tilde{\mathbf{X}}^{\prime} \mathbf{A} \widetilde{\mathbf{X}}\right)-\operatorname{det}\left(\mathbf{X}^{\prime} \mathbf{A} \mathbf{X}\right) & \geq \operatorname{det}\left(\tilde{\mathbf{X}}^{\prime} \mathbf{A} \widetilde{\mathbf{X}}\right)-(\Delta-4 \rho)^{2}(\Delta-8 \rho) \\
& =4 \rho(\Delta-4 \rho)\left(\Delta-8 \rho-\rho(1+\rho)^{2}\right)>0,
\end{aligned}
$$

because

$$
\Delta-8 \rho-\rho(1+\rho)^{2}= \begin{cases}4-\rho\left(\rho^{2}+3\right) & \text { if } \quad n=4 \\ \rho^{2}(-\rho+n-4)+\rho(2 n-11)+n & \text { if } \quad n \geq 8\end{cases}
$$

is positive for all $\rho \in(0,1)$.

Case $3 \mathbf{X}=[\mathbf{x}, \mathbf{y}, \mathbf{z}] \in C_{3}$ 
We can write $C_{3}$ as $C_{3}=C_{3.1} \cup C_{3.2} \cup C_{3.3}$, where

$C_{3.1}=\left\{[\boldsymbol{\alpha}, \boldsymbol{\beta}, \boldsymbol{\gamma}] \in M_{n \times 3}( \pm 1): \operatorname{cons}(\boldsymbol{\alpha})=0, \operatorname{cons}(\boldsymbol{\beta})=1, \operatorname{cons}(\boldsymbol{\gamma})=2\right\}$,

$C_{3.2}=\left\{[\boldsymbol{\alpha}, \boldsymbol{\beta}, \boldsymbol{\gamma}] \in M_{n \times 3}( \pm 1): \operatorname{cons}(\boldsymbol{\alpha})=0, \operatorname{cons}(\boldsymbol{\beta}) \geq 2, \operatorname{cons}(\boldsymbol{\gamma}) \geq 2\right\}$,

$C_{3.3}=\left\{[\boldsymbol{\alpha}, \boldsymbol{\beta}, \boldsymbol{\gamma}] \in M_{n \times 3}( \pm 1): \operatorname{cons}(\boldsymbol{\alpha})=0, \operatorname{cons}(\boldsymbol{\beta})=1, \operatorname{cons}(\boldsymbol{\gamma}) \geq 3\right\}$.

In this case, the proof follows from the following subcases.

Subcase 3.1 $\mathbf{X}=[\mathbf{x}, \mathbf{y}, \mathbf{z}] \in C_{3.1}$. Let $a=\operatorname{fcons}(\mathbf{z})$ and $b=\operatorname{scons}(\mathbf{z})$. From Lemma 3, we have

$$
\operatorname{det}\left(\mathbf{X}^{\prime} \mathbf{A X}\right)=\operatorname{det}\left([\mathbf{x}, \mathbf{y}, \mathbf{z}]^{\prime} \mathbf{A}[\mathbf{x}, \mathbf{y}, \mathbf{z}]\right)=\operatorname{det}\left([\mathbf{x}, \mathbf{z}, \mathbf{y}]^{\prime} \mathbf{A}[\mathbf{x}, \mathbf{z}, \mathbf{y}]\right)
$$

From equality (10), Fischer's inequality and Lemma 4, we obtain

$$
\operatorname{det}\left(\mathbf{X}^{\prime} \mathbf{A X}\right) \leq(\Delta-4 \rho)\left[\Delta(\Delta-8 \rho)-\left(\mathbf{x}^{\prime} \mathbf{A z}\right)^{2}\right]
$$

By Lemmas 5(b) and 6, we conclude that

$$
\left(\mathbf{x}^{\prime} \mathbf{A z}\right)^{2}=\left((n+2 a-2 b-2)(1+\rho)^{2}+2(1+\rho)\right)^{2} \geq 4 \rho^{2}(1+\rho)^{2},
$$

since $n+2 a-2 b-2$ is even. By (11) and (12), it follows that

$$
\begin{aligned}
\operatorname{det}\left(\widetilde{\mathbf{X}}^{\prime} \mathbf{A} \widetilde{\mathbf{X}}\right)-\operatorname{det}\left(\mathbf{X}^{\prime} \mathbf{A X}\right) \geq & \operatorname{det}\left(\widetilde{\mathbf{X}}^{\prime} \mathbf{A} \widetilde{\mathbf{X}}\right) \\
& -(\Delta-4 \rho)\left[\Delta(\Delta-8 \rho)-\left(\mathbf{x}^{\prime} \mathbf{A z}\right)^{2}\right] \\
= & (\Delta-4 \rho)(1+\rho)^{2} \\
& \cdot\left[((n+2 a-2 b-2)(1+\rho)+2)^{2}-4 \rho^{2}\right] \geq 0 .
\end{aligned}
$$

Subcase 3.2 $\mathbf{X}=[\mathbf{x}, \mathbf{y}, \mathbf{z}] \in C_{3.2}$. From Hadamard's inequality and Lemma 4, we see that

$$
\operatorname{det}\left(\mathbf{X}^{\prime} \mathbf{A X}\right) \leq\left(\mathbf{x}^{\prime} \mathbf{A x}\right)\left(\mathbf{y}^{\prime} \mathbf{A y}\right)\left(\mathbf{z}^{\prime} \mathbf{A z}\right) \leq \Delta(\Delta-8 \rho)^{2}
$$

Hence

$$
\begin{aligned}
\operatorname{det}\left(\widetilde{\mathbf{X}}^{\prime} \mathbf{A} \widetilde{\mathbf{X}}\right)-\operatorname{det}\left(\mathbf{X}^{\prime} \mathbf{A} \mathbf{X}\right) & \geq \operatorname{det}\left(\widetilde{\mathbf{X}}^{\prime} \mathbf{A} \widetilde{\mathbf{X}}\right)-\Delta(\Delta-8 \rho)^{2} \\
& =4 \rho\left[\Delta(\Delta-8 \rho)-\rho(1+\rho)^{2}(\Delta-4 \rho)\right] \\
& \geq 4 \rho\left[(\Delta-4 \rho)(\Delta-8 \rho)-\rho(1+\rho)^{2}(\Delta-4 \rho)\right] \\
& =4 \rho(\Delta-4 \rho)\left[(n-2-\rho)(1+\rho)^{2}+2-6 \rho\right] .
\end{aligned}
$$

Since $n=4,8, \ldots$ and $\rho \in(0,1)$, we obtain $(n-2-\rho)(1+\rho)^{2}+2-6 \rho \geq(4-2-\rho)(1+\rho)^{2}+2-6 \rho=4-3 \rho-\rho^{3}>0$.

Therefore $\operatorname{det}\left(\widetilde{\mathbf{X}}^{\prime} \mathbf{A} \widetilde{\mathbf{X}}\right)>\operatorname{det}\left(\mathbf{X}^{\prime} \mathbf{A} \mathbf{X}\right)$. 
Subcase 3.3 $\mathbf{X}=[\mathbf{x}, \mathbf{y}, \mathbf{z}] \in C_{3.3}$. Similarly, we conclude from Hadamard's inequality and Lemma 4 that

$$
\operatorname{det}\left(\mathbf{X}^{\prime} \mathbf{A X}\right) \leq\left(\mathbf{x}^{\prime} \mathbf{A} \mathbf{x}\right)\left(\mathbf{y}^{\prime} \mathbf{A y}\right)\left(\mathbf{z}^{\prime} \mathbf{A z}\right) \leq \Delta(\Delta-4 \rho)(\Delta-12 \rho)
$$

Therefore

$$
\begin{aligned}
\operatorname{det}\left(\widetilde{\mathbf{X}}^{\prime} \mathbf{A} \widetilde{\mathbf{X}}\right)-\operatorname{det}\left(\mathbf{X}^{\prime} \mathbf{A X}\right) & \geq \operatorname{det}\left(\widetilde{\mathbf{X}}^{\prime} \mathbf{A} \widetilde{\mathbf{X}}\right)-\Delta(\Delta-4 \rho)(\Delta-12 \rho) \\
& =4 \rho(1+\rho)(\Delta-4 \rho)\left[n-\rho^{2}+(n-3) \rho\right]>0,
\end{aligned}
$$

since $\rho \in(0,1)$. The proof is complete.

Proof of Theorem 2 The D-optimality of the design with the design matrix $\widetilde{\mathbf{X}}$ given by (3), when $\rho=0$, follows by the same argument as in the beginning of the proof of Theorem 1 . So, we assume that $\rho \in(0,1 /(n-2)]$ and (by Lemma 3$)$ the first row of $\mathbf{X}$ contains all ones. Therefore, we can consider the class of the design matrices $C_{1} \cup C_{2} \cup C_{3} \cup C_{4}$, where $C_{1}, C_{2}, C_{3}$ are described in Theorem 1 and

$$
C_{4}=\left\{[\boldsymbol{\alpha}, \boldsymbol{\beta}, \boldsymbol{\gamma}] \in M_{n \times 3}( \pm 1): \operatorname{cons}(\boldsymbol{\alpha})=0, \operatorname{cons}(\boldsymbol{\beta})=1, \operatorname{cons}(\boldsymbol{\gamma})=1\right\}
$$

The proof is similar to that in Theorem 1. From that theorem, it follows that $\operatorname{det}\left(\widetilde{\mathbf{X}}^{\prime} \mathbf{A} \widetilde{\mathbf{X}}\right) \geq \operatorname{det}\left(\mathbf{X}^{\prime} \mathbf{A X}\right)$ if $\mathbf{X}=[\mathbf{x}, \mathbf{y}, \mathbf{z}] \in C_{1} \cup C_{2} \cup C_{3}$. Assume that $\mathbf{X}=[\mathbf{x}, \mathbf{y}, \mathbf{z}] \in$ $C_{4}$. Without loss of generality we can suppose that $\operatorname{fcons}(\mathbf{y})=a>b=\operatorname{fcons}(\mathbf{z})$. From Lemmas 5(a), (c) and 4, we obtain

$$
\operatorname{det}\left(\mathbf{X}^{\prime} \mathbf{A X}\right)=\operatorname{det}\left[\begin{array}{ccc}
\Delta & \Psi_{1} & \Psi_{2} \\
\Psi_{1} & \Delta-4 \rho & \Psi_{3} \\
\Psi_{2} & \Psi_{3} & \Delta-4 \rho
\end{array}\right],
$$

where

$$
\begin{gathered}
\Psi_{1}=(2 a-n)(1+\rho)^{2}, \\
\Psi_{2}=(2 b-n)(1+\rho)^{2}, \\
\Psi_{3}=(n-2 a+2 b-2)(1+\rho)^{2}+2(1+\rho) .
\end{gathered}
$$

We must divide the proof into two cases.

Case 1 If $a \neq \frac{n}{2}$, then from Fischer's inequality, it follows that

$$
\operatorname{det}\left(\mathbf{X}^{\prime} \mathbf{A X}\right) \leq(\Delta-4 \rho)\left[\Delta(\Delta-4 \rho)-\Psi_{1}^{2}\right] \leq(\Delta-4 \rho)\left[\Delta(\Delta-4 \rho)-4(1+\rho)^{4}\right]
$$

and

$$
\begin{aligned}
\operatorname{det}\left(\tilde{\mathbf{X}}^{\prime} \mathbf{A} \widetilde{\mathbf{X}}\right)-\operatorname{det}\left(\mathbf{X}^{\prime} \mathbf{A} \mathbf{X}\right) & \geq \operatorname{det}\left(\tilde{\mathbf{X}}^{\prime} \mathbf{A} \widetilde{\mathbf{X}}\right)-(\Delta-4 \rho)\left[\Delta(\Delta-4 \rho)-4(1+\rho)^{4}\right] \\
& =(\Delta-4 \rho)\left[-4 \rho \Delta-4 \rho^{2}(1+\rho)^{2}+4(1+\rho)^{4}\right] .
\end{aligned}
$$


It is clear that $\Delta \leq n(1+\rho)^{2}$. Consequently

$$
\begin{aligned}
\operatorname{det}\left(\widetilde{\mathbf{X}}^{\prime} \mathbf{A} \widetilde{\mathbf{X}}\right)-\operatorname{det}\left(\mathbf{X}^{\prime} \mathbf{A X}\right) & \geq(\Delta-4 \rho)\left[-4 \rho n(1+\rho)^{2}-4 \rho^{2}(1+\rho)^{2}+4(1+\rho)^{4}\right] \\
& =4(\Delta-4 \rho)(1+\rho)^{2}[1+\rho(2-n)] \geq 0,
\end{aligned}
$$

because $\rho \in(0,1 /(n-2)]$.

Case 2 If $a=\frac{n}{2}$, then $b \neq \frac{n}{2}$. From Lemma 3, we have

$$
\operatorname{det}\left(\mathbf{X}^{\prime} \mathbf{A} \mathbf{X}\right)=\operatorname{det}\left([\mathbf{x}, \mathbf{y}, \mathbf{z}]^{\prime} \mathbf{A}[\mathbf{x}, \mathbf{y}, \mathbf{z}]\right)=\operatorname{det}\left([\mathbf{x}, \mathbf{z}, \mathbf{y}]^{\prime} \mathbf{A}[\mathbf{x}, \mathbf{z}, \mathbf{y}]\right)
$$

and from Fischer's inequality, we conclude that

$$
\operatorname{det}\left(\mathbf{X}^{\prime} \mathbf{A X}\right) \leq(\Delta-4 \rho)\left[\Delta(\Delta-4 \rho)-\Psi_{2}^{2}\right] \leq(\Delta-4 \rho)\left[\Delta(\Delta-4 \rho)-4(1+\rho)^{4}\right]
$$

According to Case 1 we have $\operatorname{det}\left(\widetilde{\mathbf{X}}^{\prime} \mathbf{A} \widetilde{\mathbf{X}}\right) \geq \operatorname{det}\left(\mathbf{X}^{\prime} \mathbf{A X}\right)$ and the proof is complete.

Proof of Theorem 3 If $\rho=0$, then the theorem is true by Hadamard (1893). So, assume that $\rho \in(0,1 /(n-2))$.

$(\Leftarrow)$ It is clear, because $\operatorname{det}\left(\mathbf{X}_{*}^{\prime} \mathbf{A} \mathbf{X}_{*}\right)=\operatorname{det}\left(\tilde{\mathbf{X}}^{\prime} \mathbf{A} \widetilde{\mathbf{X}}\right)$, where $\widetilde{\mathbf{X}}$ is given by (3) and it is a D-optimal design by Theorem 2 .

$(\Rightarrow)$ Let $\mathbf{X}_{*}=\left[\mathbf{x}_{*}, \mathbf{y}_{*}, \mathbf{z}_{*}\right]$ be D-optimal design in $M_{n \times 3}( \pm 1)$. Thus from Theorem 2 , we obtain $\operatorname{det}\left(\mathbf{X}_{*}^{\prime} \mathbf{A} \mathbf{X}_{*}\right)=\Delta(\Delta-4 \rho)(\Delta-8 \rho)-4 \rho^{2}(1+\rho)^{2}(\Delta-4 \rho)$. From the proofs of Theorems 1 and 2, we have $\operatorname{det}\left(\mathbf{X}_{*}^{\prime} \mathbf{A} \mathbf{X}_{*}\right)>\operatorname{det}\left(\mathbf{X}^{\prime} \mathbf{A X}\right)$ for each $\mathbf{X}$ belonging to

$$
M_{n \times 3}( \pm 1) \backslash\left\{[\boldsymbol{\alpha}, \boldsymbol{\beta}, \boldsymbol{\gamma}] \in M_{n \times 3}( \pm 1): \operatorname{cons}(\boldsymbol{\alpha})=0, \operatorname{cons}(\boldsymbol{\beta})=1, \operatorname{cons}(\boldsymbol{\gamma})=2\right\}
$$

Now, let $\mathbf{X}_{*} \in\left\{[\boldsymbol{\alpha}, \boldsymbol{\beta}, \boldsymbol{\gamma}] \in M_{n \times 3}( \pm 1): \operatorname{cons}(\boldsymbol{\alpha})=0, \operatorname{cons}(\boldsymbol{\beta})=1, \operatorname{cons}(\boldsymbol{\gamma})=2\right\}$. Then from Lemma 4, it follows that $\mathbf{x}_{*}^{\prime} \mathbf{A} \mathbf{x}_{*}=\Delta, \mathbf{y}_{*}^{\prime} \mathbf{A} \mathbf{y}_{*}=\Delta-4 \rho, \mathbf{z}_{*}^{\prime} \mathbf{A} \mathbf{z}_{*}=\Delta-8 \rho$. By Lemma 3, we obtain

$$
\operatorname{det}\left(\mathbf{X}_{*}^{\prime} \mathbf{A} \mathbf{X}_{*}\right)=\operatorname{det}\left(\left[\mathbf{x}_{*}, \mathbf{z}_{*}, \mathbf{y}_{*}\right]^{\prime} \mathbf{A}\left[\mathbf{x}_{*}, \mathbf{z}_{*}, \mathbf{y}_{*}\right]\right)
$$

Applying Fischer's inequality, Lemmas 5(b) and 6, we can write

$$
\begin{aligned}
\operatorname{det}\left(\mathbf{X}_{*}^{\prime} \mathbf{A} \mathbf{X}_{*}\right) & \leq(\Delta-4 \rho)\left[\Delta(\Delta-8 \rho)-\left(\mathbf{x}_{*}^{\prime} \mathbf{A} \mathbf{z}_{*}\right)^{2}\right] \\
& \leq(\Delta-4 \rho)\left[\Delta(\Delta-8 \rho)-4 \rho^{2}(1+\rho)^{2}\right] .
\end{aligned}
$$

By assumption, $\mathbf{X}_{*}$ is D-optimal design, so in inequalities (15) and (16) must hold equalities. From Fischer's inequality, it follows that the equality in (15) holds if and only if $\mathbf{x}_{*}^{\prime} \mathbf{A} \mathbf{y}_{*}=\mathbf{y}_{*}^{\prime} \mathbf{A} \mathbf{z}_{*}=0$. In (16), the equality holds if and only if $\mathbf{x}_{*}^{\prime} \mathbf{A} \mathbf{z}_{*}=$ $\pm 2 \rho(1+\rho)$. So the matrix $\mathbf{X}_{*}^{\prime} \mathbf{A} \mathbf{X}_{*}$ has the form (4). 
Proof of Theorem $4(\Leftarrow)$ By Lemmas 4 and 5, the matrix $\mathbf{X}_{*}^{\prime} \mathbf{A} \mathbf{X}_{*}$ has the form (4). Therefore, from Theorem 3 the design $\mathbf{X}_{*}$ is D-optimal.

$(\Rightarrow)$ By the assumption and Theorem 3 the matrix $\mathbf{X}_{*}^{\prime} \mathbf{A} \mathbf{X}_{*}$ has the form (4). From Lemma 4, it follows that $\mathbf{x}_{*}^{\prime} \mathbf{A} \mathbf{x}_{*}=\Delta \Leftrightarrow \operatorname{cons}\left(\mathbf{x}_{*}\right)=0, \mathbf{y}_{*}^{\prime} \mathbf{A} \mathbf{y}_{*}=\Delta-4 \rho \Leftrightarrow$ $\operatorname{cons}\left(\mathbf{y}_{*}\right)=1, \mathbf{z}_{*}^{\prime} \mathbf{A} \mathbf{z}_{*}=\Delta-8 \rho \Leftrightarrow \operatorname{cons}\left(\mathbf{z}_{*}\right)=2$. Let $a=$ fcons $\left(\mathbf{y}_{*}\right), b=$ fcons $\left(\mathbf{z}_{*}\right), c=\operatorname{scons}\left(\mathbf{z}_{*}\right)$. By Lemma 5(c), we obtain $\mathbf{x}_{*}^{\prime} \mathbf{A} \mathbf{y}_{*}= \pm(2 a-n)(1+\rho)^{2}=0$ and hence $a=\frac{n}{2}$. Therefore, from Lemma 5(b), (d), we conclude that $\mathbf{x}_{*}^{\prime} \mathbf{A} \mathbf{z}_{*}=$ $\pm(1+\rho)((n+2 b-2 c-2)(1+\rho)+2)= \pm 2 \rho(1+\rho) \Rightarrow c-b=\frac{n}{2}$, and $\mathbf{y}_{*}^{\prime} \mathbf{A} \mathbf{z}_{*}= \pm 2(b+c-n)(1+\rho)^{2}=0 \Rightarrow b+c=n$. Hence $b=\frac{n}{4}, c=\frac{3 n}{4}$. The theorem is proved.

Proof of Theorem 5 (Sketch) Assume that $\mathbf{X}^{*}=\left[\mathbf{x}^{*}, \mathbf{y}^{*}, \mathbf{z}^{*}\right]$ is the matrix of the D-optimal weighing design for three objects. By Theorem 4 , it follows that cons $\left(\mathbf{x}^{*}\right)=$ $0, \operatorname{cons}\left(\mathbf{y}^{*}\right)=1, \operatorname{cons}\left(\mathbf{z}^{*}\right)=2$ and fcons $\left(\mathbf{y}^{*}\right)=\frac{n}{2}, \operatorname{fcons}\left(\mathbf{z}^{*}\right)=\frac{n}{4}, \operatorname{scons}\left(\mathbf{z}^{*}\right)=\frac{3 n}{4}$ exact to permuting columns of the matrix $\mathbf{X}^{*}$. It is easy to calculate that $\mathbf{1}^{\prime} \mathbf{A} \mathbf{1}=$ $(n-2)(1-\rho)^{2}+2(1-\rho), \mathbf{1}^{\prime} \mathbf{A} \mathbf{x}^{*}=0, \mathbf{1}^{\prime} \mathbf{A} \mathbf{y}^{*}= \pm 2 \rho(1-\rho), \mathbf{1}^{\prime} \mathbf{A} \mathbf{z}^{*}=0$. Hence and from Theorem 3 , we obtain

$$
\left[\mathbf{1}, \mathbf{X}^{*}\right]^{\prime} \mathbf{A}\left[\mathbf{1}, \mathbf{X}^{*}\right]=\left[\begin{array}{cccc}
\mathbf{1}^{\prime} \mathbf{A} \mathbf{1} & 0 & \pm 2 \rho(1-\rho) & 0 \\
0 & \Delta & 0 & \pm 2 \rho(1+\rho) \\
\pm 2 \rho(1-\rho) & 0 & \Delta-4 \rho & 0 \\
0 & \pm 2 \rho(1+\rho) & 0 & \Delta-8 \rho
\end{array}\right]
$$

For D-optimal factorial design with 3 two-level factors with a first-order autoregressive errors, analogous result as that in Lemma 1 is true. So to prove D-optimality of the design $\left[\mathbf{1}, \mathbf{X}^{*}\right]$, we show (similar as in the proofs of Theorems 1 and 2 ) that the determinant of the matrix given by (17) is greater or equal than $\operatorname{det}\left([\mathbf{1}, \mathbf{X}]^{\prime} \mathbf{A}[\mathbf{1}, \mathbf{X}]\right)$ for all $\mathbf{X} \in M_{n \times 3}( \pm 1)$. If $n=4$, then by Cauchy theorem and equality (17), it follows that

$$
\begin{aligned}
\operatorname{det}\left(\left[\mathbf{1}, \mathbf{X}^{*}\right]^{\prime} \mathbf{A}\left[\mathbf{1}, \mathbf{X}^{*}\right]\right) & =\operatorname{det}\left(\left[\mathbf{1}, \mathbf{X}^{*}\right]^{\prime}\right) \operatorname{det}(\mathbf{A}) \operatorname{det}\left(\left[\mathbf{1}, \mathbf{X}^{*}\right]\right) \\
& =\operatorname{det}\left(\left[\mathbf{1}, \mathbf{X}^{*}\right]^{\prime}\left[\mathbf{1}, \mathbf{X}^{*}\right]\right) \operatorname{det}(\mathbf{A}) \\
& =4^{4} \operatorname{det}(\mathbf{A}) .
\end{aligned}
$$

Hence and by Hadamard (1893) the design $\left[\mathbf{1}, \mathbf{X}^{*}\right]$ is D-optimal. Let $n \geq 8$. Now, we must consider the same cases and subcases as in proofs of Theorems 1 and 2 . In each case and subcase, we use the same inequality, but calculations are longer and more complicated. As an example, we show the proof in Subcase 3.1. Let $\mathbf{X}=[\mathbf{x}, \mathbf{y}, \mathbf{z}] \in C_{3.1}$. It is clear that

$$
\begin{aligned}
\operatorname{det}\left([\mathbf{1}, \mathbf{X}]^{\prime} \mathbf{A}[\mathbf{1}, \mathbf{X}]\right) & =\operatorname{det}\left([\mathbf{1}, \mathbf{x}, \mathbf{y}, \mathbf{z}]^{\prime} \mathbf{A}[\mathbf{1}, \mathbf{x}, \mathbf{y}, \mathbf{z}]\right) \\
& =\operatorname{det}\left([\mathbf{1}, \mathbf{y}, \mathbf{x}, \mathbf{z}]^{\prime} \mathbf{A}[\mathbf{1}, \mathbf{y}, \mathbf{x}, \mathbf{z}]\right)
\end{aligned}
$$


Hence and by Fischer's inequality, it follows that

$$
\operatorname{det}\left([\mathbf{1}, \mathbf{X}]^{\prime} \mathbf{A}[\mathbf{1}, \mathbf{X}]\right) \leq\left[\mathbf{1}^{\prime} \mathbf{A} \mathbf{1}(\Delta-4 \rho)-\left(\mathbf{1}^{\prime} \mathbf{A} \mathbf{y}\right)^{2}\right]\left[\Delta(\Delta-8 \rho)-\left(\mathbf{x}^{\prime} \mathbf{A z}\right)^{2}\right] .
$$

By the assumption that $\operatorname{cons}(\mathbf{y})=1$, the inequality $\left(\mathbf{1}^{\prime} \mathbf{A y}\right)^{2} \geq 4 \rho^{2}(1-\rho)^{2}$ holds. From inequality (12), we have $\left(\mathbf{x}^{\prime} \mathbf{A z}\right)^{2} \geq 4 \rho^{2}(1+\rho)^{2}$. Therefore

$$
\begin{aligned}
\operatorname{det}\left([\mathbf{1}, \mathbf{X}]^{\prime} \mathbf{A}[\mathbf{1}, \mathbf{X}]\right) \leq & {\left[\mathbf{1}^{\prime} \mathbf{A} \mathbf{1}(\Delta-4 \rho)-4 \rho^{2}(1-\rho)^{2}\right] } \\
& \cdot\left[\Delta(\Delta-8 \rho)-4 \rho^{2}(1+\rho)^{2}\right] \\
= & \operatorname{det}\left(\left[\mathbf{1}, \mathbf{X}^{*}\right]^{\prime} \mathbf{A}\left[\mathbf{1}, \mathbf{X}^{*}\right]\right) .
\end{aligned}
$$

The proof is complete.

\section{References}

Banerjee KS (1975) Weighing designs for chemistry, medicine, economics, operations research, statistics. Marcel Dekker Inc., New York

Cheng CS (1980) Optimality of some weighing and $2^{n}$ fractional factorial designs. Ann Stat 8:436-446

Cohn JHE (1967) On determinants with elements \pm 1 . J Lond Math Soc 42:436-442

Cohn JHE (1989) On determinants with elements \pm 1, II. Bull Lond Math Soc 21:36-42

Galil Z, Kiefer J (1980) D-optimum weighing designs. Ann Stat 8:1293-1306

Hadamard J (1893) Résolution d'une question relative aux déterminants. Bull Sci Math 2:240-246

Jacroux M, Wong CS, Masaro JC (1983) On the optimality of chemical balance weighing designs. J Stat Plann Inference 8:231-240

Katulska K, Smaga Ł (2010) On some construction of D-optimal chemical balance weighing designs. Colloquium Biometricum 40:155-164

Li CH, Yang SY (2005) On a conjecture in D-optimal designs with $n \equiv 0(\bmod 4)$. Linear Algebra Appl 400:279-290

Yeh HG, Lo Huang MN (2005) On exact D-optimal designs with 2 two-level factors and $n$ autocorrelated observations. Metrika 61:261-275 